\title{
OSTEOARTHRITIS
}

\section{Cartilage matrix stiffness regulates chondrocyte metabolism and OA pathogenesis}

Cartilage extracellular matrix (ECM) is known to undergo remodeling as osteoarthritis (OA) progresses, manifesting as loss of ECM flexibility even before cartilage destruction occurs; however, the molecular basis of this ECM stiffening is unclear. New research indicates that these mechanical alterations to the ECM promote OA pathogenesis by regulating chondrocyte metabolism via the Rho-Rho kinasemyosin light chain (Rho-ROCK-MLC) mechanotransduction pathway.

\section{4 ...inhibition of the Rho- ROCK-MLC axis reversed these stiffening-mediated metabolic effects 77}

"Osteoarthritic cartilage destruction is primarily caused by an imbalance between chondrocyte catabolism and anabolism," explains author Jang-Soo Chun.

"Various cytokines that regulate this metabolic balance have been identified and extensively studied in terms of their roles in OA pathogenesis. In the present work, we demonstrated that the biophysical properties of the chondrocyte microenvironment (that is, cartilage ECM stiffness) also serve as a critical regulator of chondrocyte metabolism and OA pathogenesis."

The researchers conducted in vitro and in vivo studies to show that ageing and mechanical stress-two major risk factors for OA-drive collagen crosslinking and cartilage ECM stiffening via distinct mechanisms: age-related accumulation of advanced glycation endproducts (AGEs) and upregulation of the collagen cross-linking enzyme lysyl oxidase (LOX), respectively. In primary chondrocyte cultures, increased substrate stiffness was associated with increased expression of matrix-degrading enzymes and reduced expression of cartilage ECM molecules, whereas inhibition of the
Rho-ROCK-MLC axis reversed these stiffening-mediated metabolic effects.

In mice, neither ribose-mediated AGE accumulation nor overexpression of LOX in knee cartilage was sufficient to induce spontaneous OA but did sensitize the joints to surgically induced mechanical instability, and intra-articular injection of Rho-ROCK-MLC inhibitors reduced damage due to post-traumatic OA.

The team suggest that modulating ECMstiffening-mediated mechanotransduction could be an effective therapeutic approach for OA. "Our results identify matrix stiffness as a key 'design parameter' for biomaterials and scaffold development in tissue engineering or cell-based $\mathrm{OA}$ treatment," Chun concludes.

\section{Sarah Onuora}

Original article Kim, J. H. et al. Matrix cross-linkingmediated mechanotransduction promotes posttraumatic osteoarthritis. Proc. Natl Acad. Sci. USA doi:10.1073/ pnas. 1505700112 